\title{
GRAVITY MODES IN ZZ CETI STARS. III. EFFECTS OF TURBULENT DISSIPATION

\author{
Peter Goldreich ${ }^{1}$ AND YANQIN Wu ${ }^{1,2}$ \\ Received 1998 October 4 ; accepted 1999 May 14
}

\begin{abstract}
We investigate dynamical interactions between turbulent convection and $g$-mode pulsations in ZZ Ceti variables. Since our understanding of turbulence is rudimentary, we are compelled to settle for order-ofmagnitude results. A key feature of these interactions is that convective response times are much shorter than pulsation periods. Thus the dynamical interactions enforce near uniform horizontal velocity inside the convection zone. They also give rise to a narrow shear layer in the region of convective overshoot at the top of the radiative interior. Turbulent damping inside the convection zone is negligible for all modes, but that in the region of convective overshoot may be significant for a few long-period modes near the red edge of the instability strip. These conclusions are in accord with those reached earlier by Brickhill. Our major new result concerns nonlinear damping arising from the Kelvin-Helmholtz instability of the aforementioned shear layer. Amplitudes of overstable modes saturate where dissipation due to this instability balances excitation by convective driving. This mechanism of amplitude saturation is most effective for long-period modes, and it may play an important role in defining the red edge of the instability strip.
\end{abstract}

Subject headings: gravitation - stars: interiors - stars: oscillations stars: variables: other (ZZ Ceti) — turbulence

\section{INTRODUCTION}

Variable white dwarfs with hydrogen atmospheres, $\mathrm{ZZ}$ Ceti stars, occupy an instability strip centered at $T_{\text {eff }} \approx$ $12,000 \mathrm{~K}$ with a width $\Delta T_{\text {eff }} \approx 10^{3} \mathrm{~K}$ (Bergeron et al. 1995). Aside from their effective temperatures, ZZ Ceti stars are ordinary DA white dwarfs. Thus their oscillation frequencies provide valuable data bearing on the internal structure and rotation of the most populous class of stellar remnants.

ZZ Ceti stars possess surface convection zones. The convective envelope is the seat of $g$-mode overstability. An important feature of convection in these stars is that the response time is much shorter than the periods of the observed modes. Thus, to a good approximation, the convection adjusts to the instantaneous pulsational state. This behavior plays an essential role in the overstability mechanism (Brickhill 1990; Gautschy, Ludwig, \& Freytag 1996; Goldreich \& Wu 1998, hereafter Paper I), which, following Brickhill, we refer to as convective driving.

Overstable modes in ZZ Ceti stars are nonradial $g$ modes. Their velocity fields are nearly horizontal. Interactions between $g$-mode velocity perturbations and turbulence are described by the nonlinear advection term in the fluid momentum equation. We model these interactions as being due to a turbulent viscosity. ${ }^{3}$ Turbulent convection acts to reduce the vertical shear of the horizontal velocity field. In so doing, it contributes to mode damping. We estimate the amount of turbulent damping both inside the convection zone and in the region of convective overshoot at the top of the radiative interior.

\footnotetext{
1 Theoretical Astrophysics, California Institute of Technology, 130-33, Pasadena, CA 91125; pmg@gps.caltech.edu.

${ }^{2}$ Astronomy Unit, School of Mathematical Sciences, Queen Mary and Westfield College, Mile End Road, London E1 4NS, England, UK; Y.Wu@qmw.ac.uk.

${ }^{3}$ We neglect the turbulent pressure, $p_{\text {turb }} \sim \rho v_{\mathrm{cv}}^{2}$, because its ratio to the gas pressure, which is of order unity at the photosphere, declines with depth proportional to $z^{-2}$.
}

It might seem excessive to devote an entire paper to these technical matters. However, they bear on important issues, such as the location of the red edge of the instability strip, the maximum periods, the saturation amplitudes of observed modes, and the nonlinear couplings of overstable modes. The rapid convective response that is an essential component of convective driving implies that turbulent convection must strongly suppress the velocity shear in the convection zone. Because of this suppression, turbulent damping in the convective envelope is inversely proportional to the magnitude of the turbulent viscosity. If this were not so, it would overwhelm convective driving, and every $g$-mode would be damped. The region of convective overshoot presents a different dynamics. Here turbulence weakens with increasing depth. As a result, turbulent damping in this region is greater than that in the convection zone proper. An important feature of the region of convective overshoot is the presence of a shear layer, across which the horizontal velocity of each $g$-mode jumps by an amount similar to the depth-integrated variation it would have undergone across an inviscid convection zone. The KelvinHelmholtz instability of this shear layer is a source of nonlinear mode damping, which limits the amplitudes of individual modes and provides a mechanism for nonlinear mode couplings.

This paper is organized as follows: In $\S 2$ we evaluate the effects of turbulent viscosity on $g$-modes, both inside the convection zone and in the region of convective overshoot at the top of the radiative interior. Then in $\S 3$ we apply these results to estimate the contributions of turbulent viscosity to mode damping. In $\S 4$ we analyze the saturation of the amplitude of an overstable mode due to the instability of the narrow shear layer that it forces at the top of the radiative interior. We conclude with a brief discussion in $\S 5$.

The current paper is the third of a series. The reader is referred to Paper I for descriptions of the properties of $g$-modes and scaling relations appropriate to turbulent convection. Only results of immediate relevance to the current investigation are quoted here. Symbols taken from Paper I 
are defined in Table 1. In this paper we rely on order-ofmagnitude reasoning and the quasi-adiabatic approximation. Those interested in numerical calculations of nonadiabatic eigenvalues and eigenfunctions are urged to consult Wu \& Goldreich.

\section{EFFECTS OF TURBULENT VISCOSITY}

This section consists of two parts. In the first we evaluate the reduction in the shear of a $g$-mode due to turbulent viscosity within the body of the convection zone. Our results confirm those obtained by Brickhill (1990), although our treatment differs from his. The analysis in the second part is more original. We demonstrate that the suppression of shear in the convection zone results in the formation of a shear layer in the region of convective overshoot.

Since motion in the outer layers of a DA white dwarf is all that concerns us, it proves convenient to adopt a planeparallel approximation. Perturbation theory is done with Lagrangian variables that are assumed to carry a time dependence $e^{-i \omega t}$ and a horizontal spatial dependence $e^{i k_{h} \cdot x}$. Gravity modes involve a mostly horizontal sloshing of fluid. Our principal concern is with the vertical derivative of the horizontal component of the displacement, $d \xi_{h} / d z$, because it is the largest component of the gradient of the displacement.

In $\S 2.1$ we calculate the magnitude of $d \xi_{h} / d z$ inside the convection zone. To begin, we estimate the magnitude that $d \xi_{h} / d z$ would have in the absence of turbulent viscosity. In the dual limit of an isentropic convection zone and a vanishing entropy perturbation, we show that $\left|d \xi_{h} / d z\right|$ would be equal to $l\left|\xi_{z}\right| / R$, which is smaller than $l\left|\xi_{h}\right| / R$. However, a real convection zone is not an isentrope; its specific entropy increases with depth. Moreover, a $g$-mode produces a specific entropy perturbation. It turns out that the latter departure from isentropy is more important than the former in enhancing the magnitude of $d \xi_{h} / d z$. Without turbulent viscosity, the entropy perturbation could induce a $\left|d \xi_{h} / d z\right|$ as large as $\xi_{h} / z_{\omega}$. However, inclusion of turbulent viscosity reduces this $\left|d \xi_{h} / d z\right|$ by a multiplicative factor $\omega t_{\mathrm{cv}} \ll 1$.

In $\S 2.2$ we examine the velocity shear in the region of convective overshoot below the base of the convection zone. The turbulent viscosity declines sharply with depth in this region. We approximate this decline by a discontinuous drop to zero viscosity. The application of appropriate boundary conditions then shows that the horizontal velocity is discontinuous at $z_{b}$. We evaluate the magnitude of this velocity jump and the associated jump in the gradient of the pressure perturbation.

\subsection{Velocity Shear in the Convection Zone}

The linearized equations of mass and momentum conservation, the latter in component form, read

$$
\begin{gathered}
\frac{\delta \rho}{\rho}=-i k_{h} \xi_{h}-\frac{d \xi_{z}}{d z} \\
\omega^{2} \xi_{h}=i k_{h}\left(\frac{p}{\rho} \frac{\delta p}{p}-g \xi_{z}\right)-\frac{f_{h}}{\rho}, \\
\omega^{2} \xi_{z}=\frac{p}{\rho} \frac{d}{d z}\left(\frac{\delta p}{p}\right)+g\left(\frac{\delta p}{p}+i k_{h} \xi_{h}\right)-\frac{f_{z}}{\rho}
\end{gathered}
$$

(see Paper I), where $f_{i}$ is the force per unit volume due to turbulent convection.

For the moment, we neglect the force due to turbulence. Then, differentiating equation (2) and substituting for $d \xi_{z} / d z$

\begin{tabular}{|c|c|}
\hline Symbol & Meaning \\
\hline$R \ldots \ldots \ldots \ldots$ & Stellar radius \\
\hline$g \ldots \ldots \ldots \ldots$ & Surface gravity \\
\hline$r \ldots \ldots \ldots \ldots$ & Radial distance from center of star \\
\hline$Z \ldots \ldots \ldots \ldots$ & Depth below photosphere \\
\hline$z_{b} \ldots \ldots \ldots \ldots$ & Depth at bottom of convection zone \\
\hline$z_{\omega} \ldots \ldots \ldots \ldots$ & Depth at top of mode's cavity, $z_{\omega} \sim \omega^{2} /\left(g k_{h}^{2}\right)$ \\
\hline$\omega \ldots \ldots \ldots \ldots$ & Radian mode frequency \\
\hline$n \ldots \ldots \ldots \ldots$ & Radial order of mode \\
\hline$l \ldots \ldots \ldots \ldots$ & Angular degree of mode \\
\hline$k_{h} \ldots \ldots \ldots \ldots$ & Horizontal wavevector, $k_{h}^{2}=l(l+1) / R^{2}$ \\
\hline$\rho \ldots \ldots \ldots \ldots$ & Mass density \\
\hline$p \ldots \ldots \ldots \ldots$ & Pressure \\
\hline$s \ldots \ldots \ldots \ldots$ & Specific entropy in units of $k_{B} / m_{p}$ \\
\hline$F \ldots \ldots \ldots \ldots$ & Energy flux \\
\hline$L \ldots \ldots \ldots \ldots$ & Luminosity, $L=4 \pi R^{2} F$ \\
\hline$c_{s} \ldots \ldots \ldots \ldots$ & Adiabatic sound speed, $c_{s}^{2}=(\partial p / \partial \rho)_{s}$ \\
\hline$\rho_{s} \ldots \ldots \ldots \ldots$ & $(\partial \ln \rho / \partial s)_{p}, \rho_{s}=-0.2$ for fully ionized hydrogen \\
\hline$\delta \ldots \ldots \ldots \ldots$ & Denotes Lagrangian perturbation \\
\hline$\xi_{h} \ldots \ldots \ldots \ldots$ & Horizontal component of displacement vector \\
\hline$\xi_{z} \ldots \ldots \ldots \ldots$ & Vertical component of displacement vector \\
\hline$v_{\mathrm{cv}} \ldots \ldots \ldots \ldots$ & Convective velocity, $v_{\mathrm{cv}} \sim(F / \rho)^{1 / 3}$ \\
\hline$t_{\mathrm{cv}} \cdots \cdots \cdots \cdots$ & Response time for convection, $t_{\mathrm{cv}} \sim z / v_{\mathrm{cv}}$ \\
\hline$v \ldots \ldots \ldots \ldots$ & Turbulent kinematic viscosity, $v \sim z v_{\mathrm{cv}}$ \\
\hline$A, B, C \ldots \ldots$ & Dimensionless constants, approximately 2,8 , and 8 for ZZ Ceti stars \\
\hline$\tau_{\text {th }} \ldots \ldots \ldots \ldots$ & Thermal constant at depth $z, t_{\mathrm{cv}} / \tau_{\mathrm{th}} \sim\left(v_{\mathrm{cv}} / c_{s}\right)^{2}$ in the convection zone \\
\hline$\tau_{b} \ldots \ldots \ldots \ldots$ & Unconventional thermal time constant at $z_{b}, \tau_{b} \approx p_{b} z_{b} / 7 \approx \tau_{\mathrm{th}} / 5$ at $z_{b}$ \\
\hline$\tau_{c} \ldots \ldots \ldots \ldots$ & Time constant of low pass filter for convection zone, $\tau_{c}=(B+C) \tau_{b}$ \\
\hline
\end{tabular}

TABLE 1

DEFINITIONS 
using equation (1), we obtain

$$
\frac{d \xi_{h}}{d z}=\frac{i k_{h}}{\omega^{2}}\left[\frac{d}{d z}\left(\frac{p}{\rho} \frac{\delta p}{p}\right)+g\left(\frac{\delta \rho}{\rho}+i k_{h} \xi_{h}\right)\right] .
$$

With the aid of equation (3) and the equation of state,

$$
\frac{\delta \rho}{\rho}=\frac{p}{c_{s}^{2} \rho} \frac{\delta p}{p}+\rho_{s} \delta s,
$$

we recast equation (4) in a more revealing form:

$$
\frac{d \xi_{h}}{d z}=i k_{h} \xi_{z}-\frac{i g k_{h} \rho_{s}}{\omega^{2}}\left[\frac{p}{g \rho} \frac{d s}{d z}\left(\frac{\delta p}{p}\right)-\delta s\right] .
$$

We can think of the $\delta p / p$ and $\delta s$ terms as providing adiabatic and nonadiabatic forcing of $d \xi_{h} / d z$. Both terms inside the brackets in equation (6) vanish for adiabatic perturbations in an isentropic convection zone. In their absence, the perturbations are irrotational, so $d \xi_{h} / d z=i k_{h} \xi_{z} \cdot{ }^{4}$ This value of shear would lead to negligible turbulent damping. To estimate $d \xi_{h} / d z$ when the flow is rotational, we relate $\delta p / p$ and $\delta s$ to $\xi_{h}$. Here we appeal to Paper I, which establishes that for $z \lesssim z_{b}$ and $z_{b} \lesssim z_{\omega},{ }^{5}$

$$
\frac{\delta p}{p} \approx-i k_{h} \xi_{h}
$$

and

$$
\delta s \approx \frac{A(B+C)}{1-i \omega \tau_{c}}\left(\frac{\delta p}{p}\right) .
$$

The ratio of adiabatic to nonadiabatic forcing, as estimated at $z=z_{b}$, is given by

$$
\begin{aligned}
\left(\frac{p}{g \rho} \frac{d s}{d z}\right)_{b}\left|\frac{\delta p}{p}\right|_{b} \frac{1}{|\delta s|_{b}} & \sim\left(\frac{p}{g \rho} \frac{d s}{d z}\right)_{b} \frac{\left[1+\left(\omega \tau_{c}\right)^{2}\right]^{1 / 2}}{A(B+C)} \\
& \sim\left[\left(\frac{v_{\mathrm{cv}}}{c_{s}}\right)^{4}+\left(\omega t_{\mathrm{cv}}\right)^{2}\right]_{b}^{1 / 2} \ll 1,
\end{aligned}
$$

where we make use of the relations that $g d s / d z \sim\left(v_{\mathrm{cv}} / c_{s}\right)^{2}$ and $t_{\mathrm{cv}} / \tau_{b} \sim\left(v_{\mathrm{cv}} / c_{s}\right)^{2}$. This establishes nonadiabatic forcing as the principal driver of velocity shear in the convection zone. In an inviscid convection zone, $\left|d \ln \xi_{h} / d z\right|$ would be of order $z_{\omega}^{-1}$. Acting on a shear of this magnitude, turbulent damping would overwhelm convective driving. ${ }^{6}$

Next we assess the effects of turbulence on the shear. Given our present understanding of turbulence, about the best we can do is to model the turbulent Reynolds stress by analogy with the stress due to molecular viscosity in a Newtonian fluid. The viscous force per unit volume is given by

$$
f_{i}=\frac{\partial \sigma_{i j}}{\partial x^{j}},
$$

where the stress tensor, $\sigma_{i j}$, is related to the $g$-mode's displacement field by two scalar coefficients, the shear viscosity, $v$, and the bulk viscosity, $\zeta$, according to

$$
\sigma_{i j}=-i \omega \rho\left\{v\left[\left(\frac{\partial \xi_{i}}{\partial x^{j}}+\frac{\partial \xi_{j}}{\partial x^{i}}\right)-\frac{2}{3} \frac{\partial \xi_{k}}{\partial x^{k}} \delta_{i j}\right]+\zeta \frac{\partial \xi_{k}}{\partial x^{k}} \delta_{i j}\right\} .
$$

\footnotetext{
${ }^{4}$ This is a linearized version of Kelvin's circulation theorem.

${ }^{5}$ Overstable modes have $z_{\omega}>z_{b}$. These are the only ones that concern us in this paper.

${ }^{6}$ A similar conclusion is arrived at by Brickhill (1990).
}

When the effects of turbulent viscosity are neglected, $d \xi_{h} / d z$ is by far the largest component of the gradient of the displacement. Since this term contributes to the shear but not to the divergence, we need not consider terms involving the bulk viscosity.

Turbulent viscosity has a profound effect on the horizontal component of the momentum equation (eq. [2]). The dominant term in $f_{h}$ takes the form

$$
f_{h} \approx-i \omega \frac{d}{d z}\left(\rho v \frac{d \xi_{h}}{d z}\right) .
$$

With the advantage of foresight (see eq. [16]), we know that $d \xi_{h} / d z$ increases with depth as $t_{\mathrm{cv}} \propto z^{3 / 2}$ times a factor that varies on scale $z_{\omega}>z$. Thus, carrying out the differentiation in equation (12) yields

$$
f_{h} \sim-i \omega \rho v_{\mathrm{cv}} \frac{d \xi_{h}}{d z} .
$$

Adding the approximate expression for $-f_{h} / \rho$ to the righthand side of equation (2), we arrive at

$$
\omega^{2} \xi_{h} \approx i k_{h}\left(\frac{p}{\rho} \frac{\delta p}{p}-g \xi_{z}\right)+\frac{i \omega z}{t_{\mathrm{cv}}} \frac{d \xi_{h}}{d z} .
$$

We note that the viscous term in equation (14) is approximately proportional to $z$. Hence, differentiating this equation with respect to $z$ and repeating the steps that led from equation (2) to equation (6), we obtain

$$
\frac{d \xi_{h}}{d z} \sim i k_{h} \xi_{z}-\frac{i g k_{h} \rho_{s}}{\omega^{2}}\left[\frac{p}{g \rho} \frac{d s}{d z}\left(\frac{\delta p}{p}\right)-\delta s\right]+\frac{i \omega}{t_{\mathrm{cv}}} \frac{d \xi_{h}}{d z} .
$$

Combining the terms proportional to $d \xi_{h} / d z$ leads to the expression we are after, namely,

$$
\frac{d \xi_{h}}{d z} \sim \frac{-\omega t_{\mathrm{cv}}}{1+i \omega t_{\mathrm{cv}}}\left\{k_{h} \xi_{z}-\frac{g k_{h} \rho_{s}}{\omega^{2}}\left[\frac{p}{g \rho} \frac{d s}{d z}\left(\frac{\delta p}{p}\right)-\delta s\right]\right\} .
$$

Note that equation (16) reduces to equation (6) in the limit $\omega t_{\mathrm{cv}} \gg 1$. However, since $\omega t_{\mathrm{cv}} \ll 1$, turbulent viscosity significantly reduces $d \xi_{h} / d z$.

For completeness, we provide an estimate for $d \xi_{z} / d z$. Combining equations (1), (5), (7), and (8) yields

$$
\frac{d \xi_{z}}{d z} \sim-i k_{h} \xi_{h}
$$

which is independent of the magnitude of the turbulent viscosity. The small value of $d \xi_{z} / d z$ ensures that its associated mode damping is negligible, as are the contributions from $i k_{h} \xi_{h}$ and $i k_{h} \xi_{z}$.

\subsection{Shear Layer at the Base of the Convection Zone}

Here we show that there is a shear layer at the top of the radiative interior, across which the horizontal displacement jumps by an amount similar to the depth-integrated change it would undergo in an inviscid convection zone.

We assume that the turbulent viscosity drops discontinuously to zero across the boundary at $z=z_{b}$. Then the 
continuity of the tangential stress demands that ${ }^{7}$

$$
\left.\frac{d \xi_{h}}{d z}\right|_{b^{-}}=0
$$

Next we multiply equation (2) by $\rho$ and integrate the resulting expression from the top of the stellar atmosphere to the bottom of the convection zone, taking $f_{h}$ from equation (12). This procedure yields

$$
\omega^{2} \int_{0}^{z_{b}} d z \rho \xi_{h} \approx i k_{h} \int_{0}^{z_{b}} d z \rho\left[\frac{p}{\rho}\left(\frac{\delta p}{p}\right)-g \xi_{z}\right] .
$$

We transform the right-hand side of equation (19) in two steps. Integrating by parts and using the hydrostatic equilibrium equation, $d p / d z=g \rho$, we obtain

$$
\begin{aligned}
\omega^{2} \int_{0}^{z_{b}} d z \rho \xi_{h} \approx & \frac{i k_{h} p_{b}}{g}\left[\frac{p}{\rho}\left(\frac{\delta p}{p}\right)-g \xi_{z}\right]_{b^{-}} \\
& -\frac{i k_{h}}{g} \int_{0}^{z_{b}} d z p \frac{d}{d z}\left[\frac{p}{\rho}\left(\frac{\delta p}{p}\right)-g \xi_{z}\right] .
\end{aligned}
$$

Then using equations (1), (2), and (5), and taking into account the continuity of $\delta p$ and $\xi_{z}$ across $z_{b}$, we arrive at ${ }^{8}$

$$
\begin{aligned}
\omega^{2} \int_{0}^{z_{b}} d z \rho \xi_{h} \approx & \left.\frac{\omega^{2} p_{b}}{g} \xi_{h}\right|_{b^{+}} \\
& +i k_{h} \int_{0}^{z_{b}} d z p \rho_{s}\left[\frac{p}{g \rho} \frac{d s}{d z}\left(\frac{\delta p}{p}\right)-\delta s\right] .
\end{aligned}
$$

Since, for $\omega t_{\mathrm{cv}} \ll 1, \xi_{h}$ is nearly constant within the convection zone, we establish the discontinuity of $\xi_{h}$ across $z_{b}$ to be

$$
\left.\Delta \xi_{h} \equiv \xi_{h}\right|_{b^{-}} ^{b^{+}} \approx \frac{-i g k_{h}}{\omega^{2} p_{b}} \int_{0}^{z_{b}} d z p \rho_{s}\left[\frac{p}{g \rho} \frac{d s}{d z}\left(\frac{\delta p}{p}\right)-\delta s\right] .
$$

Equation (22) merits a few comments. It may be simplified by taking advantage of the near constancy of $\delta p / p$ and $\delta s$ inside the convection zone. Both are nearly constant because of the rapid mixing by turbulent convection that occurs on the timescale $t_{\mathrm{cv}}$. Since the term involving the entropy perturbation dominates that due to the unperturbed entropy gradient, and from equation (8) $|\delta s| \sim$ $|\delta p / p| \sim\left|k_{h} \xi_{h}\right|$, we know that the fractional change in $\xi_{h}$ across $z_{b}$ is of order $z_{b} / z_{\omega}$. Thus the relative size of the jump is greatest for long-period modes in cool stars. Finally, as advertised, comparison with equation (6) reveals that the discontinuity in $\xi_{h}$ is closely related to the total variation that $\xi_{h}$ would experience within the convection zone in the absence of turbulent viscosity.

For reference, it follows directly from equation (3) that

$$
\left.\frac{p}{g \rho} \frac{d}{d z}\left(\frac{\delta p}{p}\right)\right|_{b^{-}} ^{b^{+}} \approx-\left.i k_{h} \xi_{h}\right|_{b^{-}} ^{b^{+}} .
$$

\footnotetext{
${ }^{7}$ We denote by $b^{\mp}$ quantities evaluated on the convective (minus) and radiative sides (plus) of the boundary, respectively.

${ }^{8}$ We ignore the effects of turbulent viscosity in the vertical component of the momentum equation (eq. [3]). It can be easily shown that both $-f_{z} /(g \rho)$ and $\omega^{2} \xi_{z} / g$ are much smaller than either $\delta p / p$ or $i k_{h} \xi_{h}$.
}

\section{TURBULENT DAMPING}

Damping due to turbulent viscosity is evaluated inside the convection zone in $\S 3.1$ and in the region of convective overshoot in $\S 3.2 .{ }^{9}$ The former is shown to be negligible for all modes. However, the latter may stabilize low-frequency modes, which would otherwise be overstable.

\subsection{Inside the Convection Zone}

Damping due to turbulent viscosity within the convection zone may be estimated as

$$
\gamma_{\text {vis }} \approx-\frac{\omega^{2} R^{2}}{2} \int_{0}^{z_{b}} d z \rho v\left(\frac{d \xi_{h}}{d z}\right)^{2} .
$$

Evaluating this integral with only the (dominant) entropy perturbation term from equation (8) retained on the righthand side of equation (16), we obtain

$$
\gamma_{\mathrm{vis}-\mathrm{cv}} \sim-0.3 \frac{\omega^{2} t_{\mathrm{cv}} \tau_{c}}{1+\left(\omega \tau_{c}\right)^{2}}\left(\frac{z_{b}}{z_{\omega}}\right) L\left(\frac{\delta p}{p}\right)_{b}^{2},
$$

where $t_{\mathrm{cv}}$ is evaluated at $z_{b}$, and the normalized $(\delta p / p)_{b}^{2} \sim$ $1 /\left(n L \tau_{\omega}\right)$. In deriving equation (25), we integrate over an isentropic convection zone with adiabatic index $5 / 3$ and substitute for $A, B, C, v, t_{\mathrm{cv}}$, and $v_{\mathrm{cv}}$ according to Table 1 . By contrast, radiative damping as evaluated in Paper I yields

$$
\gamma_{\mathrm{rad}} \sim-0.01 L\left(\frac{\delta p}{p}\right)_{b}^{2} .
$$

Since $\left(t_{\mathrm{cv}} / \tau_{c}\right)\left(z_{b} / z_{\omega}\right) \ll 0.03$ for overstable $g$-modes in the ZZ Ceti instability strip, turbulent damping inside the convection zone is much smaller than radiative damping.

It follows from equations (16) and (24) that turbulent damping of a given mode is maximized for $\omega t_{\mathrm{cv}} \sim 1$; it is negligible inside the convection zone because $\omega t_{\mathrm{cv}} \ll 1$ there. ${ }^{10}$ However, $\omega t_{\mathrm{cv}}$ rises with depth in the region of convective overshoot. This suggests that damping in this region may exceed that inside the convection zone.

\subsection{In the Region of Convective Overshoot}

The correction to the horizontal displacement induced by turbulent viscosity decays with depth in the radiative interior. In this subsection we study the depth dependence of this correction, which we refer to as $\tilde{\xi}_{h}$, and the turbulent damping with which it is associated.

The intensity of turbulence in the region of convective overshoot also diminishes with depth. We estimate this region's contribution to mode damping by solving a model problem. We replace the bottom of the convection zone by a horizontal plate and the region of convective overshoot by an underlying viscous fluid of uniform density whose viscosity decays exponentially with depth:

$$
v(z)=v_{b} e^{-\left(z-z_{b}\right) / \lambda}
$$

The distance below the convection zone over which turbulent mixing maintains a small subadiabatic entropy gra-

\footnotetext{
${ }^{9}$ Here we treat the region of convective overshoot as having a finite extent, unlike what we did in $\S 2.2$.

${ }^{10}$ It might seem paradoxical, but provided $\omega t_{\mathrm{cv}} \ll 1$, turbulent damping is inversely proportional to the magnitude of the turbulent viscosity. This follows because the dissipation rate is proportional to $\left(z^{2} / t_{\mathrm{cv}}\right)\left(d \xi_{h} / d z\right)^{2}$ and $\left|d \xi_{h} / d z\right| \propto \omega t_{\mathrm{cv}}$ for $\omega t_{\mathrm{cv}} \ll 1$.
} 


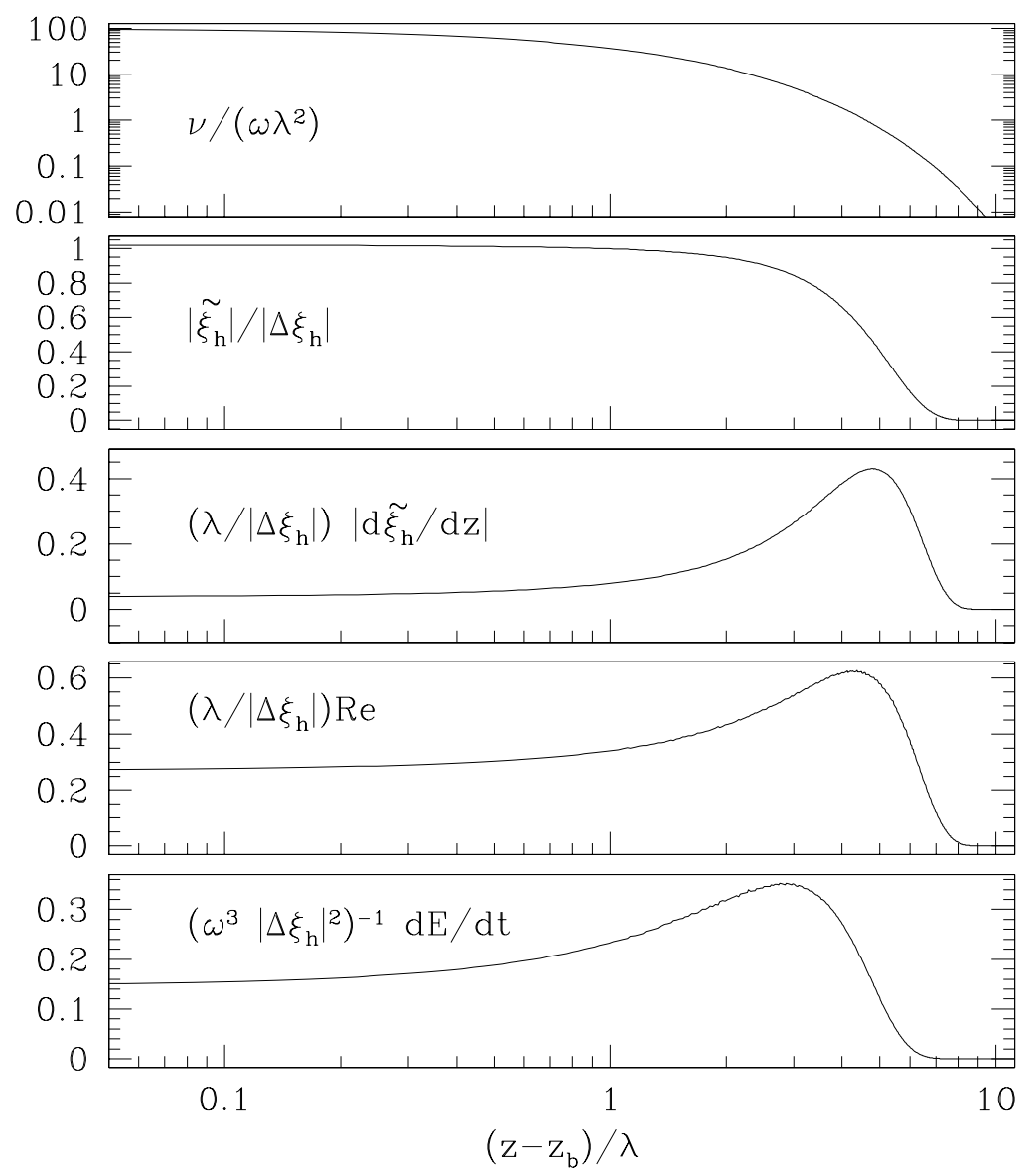

FIG. 1.-Numerical solution to the toy model in $\S 3.2$ for $\epsilon=0.1$. From top to bottom the panels display scaled dimensionless versions of the viscosity, $v$, the magnitude of the horizontal displacement, $\left|\tilde{\xi}_{h}\right|$, the magnitude of the principal component of shear, $\omega\left|d \tilde{\xi}_{h} / d z\right|$, the local Reynolds number, Re $\equiv$ $\left(\omega\left|\tilde{\xi}_{h}\right|^{2}\right) /\left(v\left|d \tilde{\xi}_{h} / d z\right|\right)$, and the viscous rate of energy dissipation per unit mass, $d E / d t \equiv v \omega^{2}\left|d \tilde{\xi}_{h} / d z\right|^{2}$, as functions of the distance below $z_{b}$ measured in units of $\lambda$. Each of the curves plotted in the bottom four panels is a function of the single variable $v /\left(\omega \lambda^{2}\right)$ and the parameter $\epsilon$. Moreover, the $\epsilon$-dependence is weak (logarithmic) for $\epsilon \ll 1$. Note that the shear, the Reynolds number, and the rate of energy dissipation all peak near the depth where $v /\left(\omega \lambda^{2}\right)=1$.

dient is of order $\lambda \ln \left(c_{s} / v_{\mathrm{cv}}\right)_{b}^{2}$, which is several times as large as $\lambda$.

The shear layer is driven by the plate, which oscillates at frequency $\omega$ with displacement amplitude $-\Delta \xi_{h}$ given by equation (22). The Navier-Stokes equation yields

$$
\frac{d}{d z}\left(v \frac{d \tilde{\xi}_{h}}{d z}\right)-i \omega \tilde{\xi}_{h}=0
$$

which is to be solved subject to the boundary conditions $\tilde{\xi}_{h}=-\Delta \xi_{h}$ at $z=z_{b}$, and $\tilde{\xi}_{h} \rightarrow 0$ as $z \rightarrow \infty$. Next we define

$$
\epsilon=\left(\frac{\omega \lambda^{2}}{v_{b}}\right)^{1 / 2} \ll 1
$$

and we change the independent variable to

$$
y=2 \epsilon e^{\left(z-z_{b}\right) / 2 \lambda-i \pi / 4}
$$

and the dependent variable to

$$
u=e^{-\left(z-z_{b}\right) / 2 \lambda} \tilde{\xi}_{h} .
$$

With these changes, equation (28) is recast in a more familiar form:

$$
\frac{d^{2} u}{d y^{2}}+\frac{1}{y} \frac{d u}{d y}+\left(1-\frac{1}{y^{2}}\right) u=0
$$

which is Bessel's equation for $n=1$. The appropriate solution, which vanishes as $|y| \rightarrow \infty$, is $H_{1}^{(2)}=J_{1}-i Y_{1}$. Here $J_{1}$ and $Y_{1}$ are Bessel functions of the first and second kind, and $H_{1}^{(2)}$ is a Hankel function of the second kind (see Abramowitz \& Stegun 1970, p. 358). The solution for $\tilde{\xi}_{h}$ may be written as

$$
\tilde{\xi}_{h}=D y H_{1}^{(2)}(y),
$$

where the coefficient $D$ is evaluated by applying the boundary condition $\tilde{\xi}_{h}=-\Delta \xi_{h}$ at $z=z_{b}$. Since $\left|y_{b}\right| \equiv\left|y\left(z_{b}\right)\right|=$ $2 \epsilon \ll 1$, we adopt the approximation $y_{b} H_{1}^{(2)}\left(y_{b}\right) \approx 2 i / \pi$, which implies

$$
D \approx \frac{i \pi \Delta \xi_{h}}{2} .
$$

The viscous stress that the plate exerts on the fluid is given by

$$
S=i \omega \rho_{b} v_{b} \frac{d \tilde{\xi}_{h}}{d z} .
$$

Making use of the identity

$$
\frac{d}{d y}\left(y H_{1}^{(2)}\right)=y H_{0}^{(2)},
$$




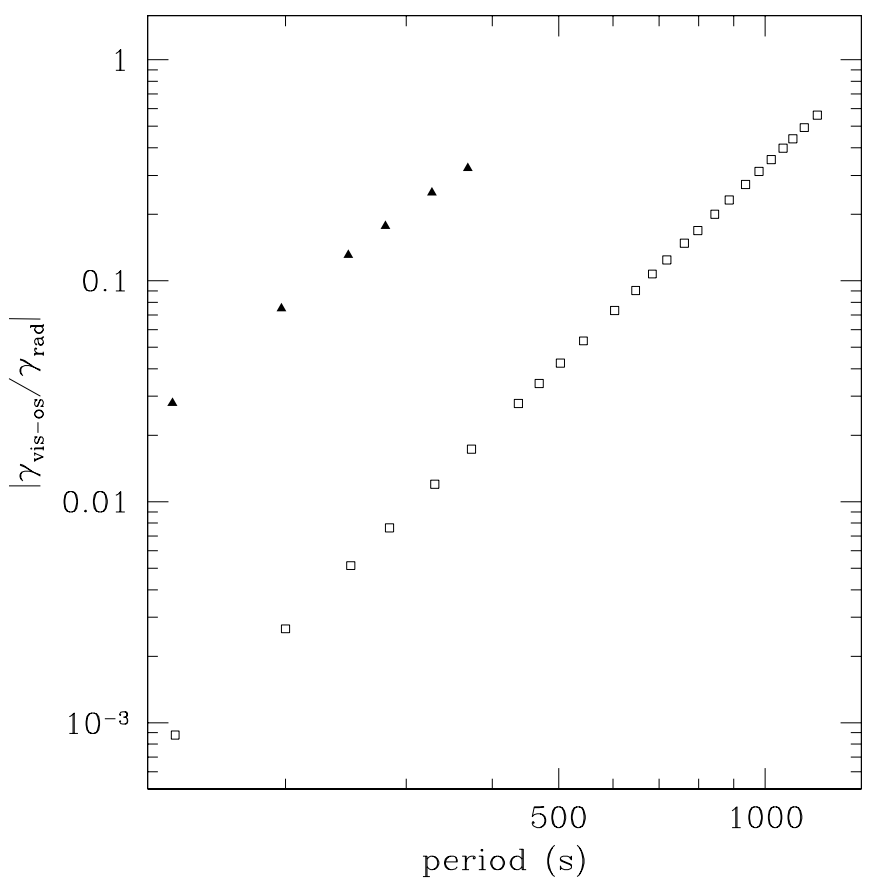

FIG. 2.-Ratio of the rate of linear turbulent damping in the overshoot region to the rate of radiative damping for two white dwarf models with $T_{\text {eff }}=12,400$ (triangles) and 12,000 K (squares), plotted against a period for $l=1$ modes. Here, $\lambda$ is taken to equal $z_{b}$ to maximize the effect of overshoot damping.

we find

$$
\begin{aligned}
S & =\frac{-\pi \omega \rho_{b} v_{b}}{4 \lambda} \Delta \xi_{h} y_{b}^{2} H_{0}^{(2)}\left(y_{b}\right) \\
& \approx 2 \omega^{2} \lambda \rho_{b} \Delta \xi_{h}\left(\ln \epsilon+\gamma+\frac{i \pi}{4}\right),
\end{aligned}
$$

where $\gamma$ is Euler's constant.

The power per unit area supplied to the fluid, $P / A$, is equal to the product of $S$ with the plate's velocity, $i \omega \Delta \xi_{h}$. To obtain the time-averaged rate at which the mode dissipates energy, we multiply $-P / A$ by $4 \pi R^{2}$ and average over both time and solid angle to arrive at

$$
\left\langle\frac{d E}{d t}\right\rangle \approx \frac{-\pi R^{2} \rho_{b} \omega^{3} \lambda\left|\Delta \xi_{h}\right|^{2}}{4}
$$

Note that $\langle d E / d t\rangle$ is independent of $v_{b}$ and proportional to $\lambda$. This is not surprising. Close to the plate the fluid moves almost rigidly, so the rate of dissipation per unit volume is small, just as it is in the interior of the convection zone. This rate first increases with depth, reaching a peak where the viscous diffusion time is of order $\omega^{-1}$, and subsequently declines. Near the peak, $v \sim \omega \lambda^{2}$, which implies a dissipation rate per unit volume $\sim \rho_{b} \omega^{3}\left|\Delta \xi_{h}\right|^{2}$. Taking the peak to have a width $\sim \lambda$ and including a factor $R^{2}$ for the integration over the area, we recover equation (38) up to a factor of order unity.

Figure 1 displays graphs of various quantities obtained from the numerical solution of the toy model that we use to estimate linear turbulent damping in the region of convective overshoot. They establish that both the shear and the turbulent dissipation reach their maximum values near the layer where $v /\left(\omega \lambda^{2}\right)=1$.
The damping rate due to convective overshoot is given by $\gamma_{\text {vis-os }}=\langle d E / d t\rangle$, since our eigenfunctions are normalized in such a way that $E=1$ (see eq. [49] of Paper I). Retaining only the (dominant) entropy perturbation in equation (22) and making use of equations (7) and (8), we find

$$
\left|\Delta \xi_{h}\right| \approx \frac{2}{35} \frac{z_{b}}{z_{\omega} k_{h}} \frac{A(B+C)}{\left[1+\left(\omega \tau_{c}\right)^{2}\right]^{1 / 2}}\left(\frac{\delta p}{p}\right)_{b} .
$$

Adopting values and relations for $A, B, C, \tau_{b}$, and $\tau_{c}$ from Table 1, we arrive at

$$
\gamma_{\mathrm{vis}-\mathrm{os}} \sim-0.2 \frac{\omega \tau_{c}}{1+\left(\omega \tau_{c}\right)^{2}}\left(\frac{\lambda}{z_{\omega}}\right) L\left(\frac{\delta p}{p}\right)_{b}^{2} .
$$

Figure 2 displays $\gamma_{\text {vis-os }} / \gamma_{\text {rad }}$ for overstable $g$-modes in stars of $T_{\text {eff }}=12,400$ and $12,000 \mathrm{~K} \cdot{ }^{11}$ We choose $\lambda / z_{b}=1$ in order to maximize turbulent damping in the region of convective overshoot. ${ }^{12}$ Even with this extreme choice for $\lambda / z_{b}$, $\gamma_{\text {vis-os }}$ is smaller than $\gamma_{\text {rad }}$ for all the overstable $g$-modes.

\section{AMPLITUDE LIMITATION DUE TO SHEAR INSTABILITY}

Kelvin-Helmholtz instability of the shear layer at the top of the radiative interior (see $\S 2.2$ ) is a source of nonlinear mode damping. As such, it acts to limit the amplitudes that overstable modes can achieve. We derive limiting amplitudes by balancing dissipation rates due to shear instability against excitation rates due to convective driving. In this section we deal with physical, as opposed to normalized, perturbations.

Even in carefully controlled laboratory settings, unstable shear layers are complex structures. Those of interest here are further complicated by externally driven turbulence associated with penetrative convection. ${ }^{13}$ In particular, turbulent mixing reduces the Brunt-Väisälä frequency, while turbulent viscosity lowers the effective Reynolds number. The former reduces stability, while the latter enhances it. It is customary to express the nonlinear stress, $\mathscr{S}$, that maintains the shear in terms of the velocity jump across the layer, $\Delta v$, and a dimensionless drag coefficient, $C_{D}$, as

$$
\mathscr{S}=\frac{1}{2} C_{D} \rho(\Delta v)^{2}
$$

(see, e.g., Landau \& Lifshitz 1976; Tritton 1977); $C_{D}$ depends only weakly on $\Delta v$ in turbulent shear layers. Terrestrial experiments indicate that $C_{D}$ varies logarithmically with the ratio of wall roughness to boundary layer width, with values as small as $10^{-3}$ being characteristic of flows over smooth walls. We might speculate that penetrative convection makes the upper boundary of the shear layer behave like a rough wall. Since we have no physical basis for assigning a reliable value to $C_{D}$, we treat it as a free parameter subject to the constraint $10^{-3} \leq C_{D} \leq 10^{-1}$.

The power per unit area input to the shear layer is $P /$ $A=\mathscr{S} \Delta v$. To obtain the time-averaged rate of energy dissipation in the shear layer, we multiply $-P / A$ by $4 \pi R^{2}$ and

\footnotetext{
${ }^{11}$ The DA white dwarf models used in this paper are provided by P. Bradley. For model details, see Bradley (1996).

${ }^{12}$ Helioseismology constrains the entire depth of the region of convective overshoot in the Sun to be less than 0.05 pressure scale heights (Basu 1997).

${ }^{13}$ We distinguish turbulence associated with convective overshoot from that due to instability of a $g$-mode's velocity shear.
} 
carry out appropriate averages over both time and solid angle. ${ }^{14}$ Next we set $\Delta v=\omega\left|\Delta \xi_{h}\right|$, where $\left|\Delta \xi_{h}\right|$ is evaluated using equation (39) as in $\S 3.2$. This series of steps leads to

$$
\left\langle\frac{d E_{\mathrm{vis}-\mathrm{n} 1}}{d t}\right\rangle \sim-0.1 C_{D} \frac{\omega \tau_{c}}{\left[\left(\omega \tau_{c}\right)^{2}+1\right]^{3 / 2}} \frac{z_{b}}{k_{h} z_{\omega}^{2}} L\left(\frac{\delta p}{p}\right)_{b}^{3} .
$$

The saturation amplitude is obtained by balancing the damping due to shear instability by the net rate of energy gain due to convective driving plus radiative damping,

$$
\left\langle\frac{d E_{\mathrm{net}}}{d t}\right\rangle \sim 0.01 \frac{\left[\left(\omega \tau_{c}\right)^{2}-1\right]}{\left[\left(\omega \tau_{c}\right)^{2}+1\right]} L\left(\frac{\delta p}{p}\right)_{b}^{2}
$$

(see Paper I). This yields

$$
\left(\frac{\delta p}{p}\right)_{b} \sim \frac{0.1}{C_{D}} \frac{\left[\left(\omega \tau_{c}\right)^{2}+1\right]^{1 / 2}\left[\left(\omega \tau_{c}\right)^{2}-1\right]}{\omega \tau_{c}} \frac{k_{h} z_{\omega}^{2}}{z_{b}} .
$$

Amplitudes of overstable modes limited by shear instability exhibit the following patterns: In a given star, $(\delta p / p)_{b}$ declines sharply with increasing period. For a fixed mode, the value of $(\delta p / p)_{b}$ rises as the host star cools. The fractional flux perturbation at the base of the convection zone is about $2(\delta p / p)_{b}$. The flux perturbation at the photosphere is smaller by the visibility reduction factor $1 /\left[1+\left(\omega \tau_{c}\right)^{2}\right]^{1 / 2}$ (see Paper I). Realistic estimates of mode amplitudes must await consideration of additional amplitude limiting processes and the calculation of nonadiabatic growth rates.

Is it safe to assume, as we have been doing, that the shear layer is turbulent? Necessary conditions are that the unperturbed shear layer has both a local shear that is large compared with $\omega$ and a local Reynolds number, Re, that is large

\footnotetext{
${ }^{14}$ The velocity jump associated with a $g$-mode shear layer varies harmonically with time and has an angular dependence given by the gradient of a spherical harmonic.
}

compared with unity. These conditions can be expressed by the dimensionless relations $\left|d \tilde{\xi}_{h} / d z\right| \gg 1$ and $\mathrm{Re}=$ $\left(\omega\left|\tilde{\xi}_{h}\right|^{2}\right) /\left(v\left|d \tilde{\xi}_{h} / d z\right|\right) \gg 1$. Based on the discussion in $\S 3.2$, we know that the left-hand side of each inequality attains a peak value $\sim\left|\Delta \xi_{h}\right| / \lambda$ near to where $v \sim \omega \lambda^{2}$ (see Fig. 1). Thus the assumption of turbulence for low $l$-modes requires $(\delta p / p)_{b} \gg\left(\lambda / z_{b}\right)\left(z_{\omega} / R\right)$. The right-hand side of this inequality is of order $z_{b} / R \approx 10^{-4}$ for the lowest frequency modes detected in stars near the red edge of the instability strip. But $(\delta p / p)_{b} \gtrsim \Delta F / F$, where $\Delta F$ is the photospheric flux variation (see Paper I). Long-period modes observed in cool ZZ Ceti stars typically exhibit fractional flux variations much greater than $10^{-4}$. Thus we conclude that these stars possess turbulent shear layers.

\section{SUMMARY}

Brickhill (1990) discussed the manner in which turbulent convection affects $g$-modes in ZZ Ceti stars. He recognized that turbulent viscosity forces the horizontal velocity to be nearly independent of depth inside the convection zone. Moreover, he deduced that turbulent damping inside the convection zone is negligible, whereas that in the region of convective overshoot might stabilize long-period modes near the red edge of the instability strip. Our investigation supports Brickhill's conclusions, except it suggests that turbulent damping by penetrative convection is, at best, of minor significance.

We extend the investigation of turbulent dissipation to include that due to the Kelvin-Helmholtz instability of the shear layer in the region of convective overshoot. Turbulence generated by this instability dissipates the mode energy at the nonlinear rate $d E / d t \propto E^{3 / 2}$. Thus the shear instability provides mechanisms for both amplitude saturation and coupling of overstable modes. We plan to investigate its consequences more thoroughly in the future.

We are indebted to Paul Bradley both for supplying us with models of DA white dwarfs and for providing a detailed review of our paper. Financial support for this research was provided by NSF grant 94-14232.

\section{REFERENCES}

Abramowitz, M., \& Stegun, I. A. 1970, Handbook of Mathematical Functions (New York: Dover)

Basu, S. 1997, MNRAS, 288, 572

Bergeron, P., Wesemael, F., Lamontagne, R., Fontaine, G., Saffer, R. A., \& Allard, N. F. 1995, ApJ, 449, 258

Bradley, P. A. 1996, ApJ, 468, 350

Brickhill, A. J. 1990, MNRAS, 246, 510
Gautschy, A., Ludwig, H. G., \& Freytag, B. 1996, A\&A, 311, 493

Goldreich, P., \& Wu, Y. 1999, ApJ, 511, 904 (Paper I)

Landau, L. D., \& Lifshitz, E. M. 1959, Fluid Mechanics (Oxford: Pergamon), 166

Tritton, D. J. 1977, Physical Fluid Dynamics (New York: van Nostrand Reinhold), 28

Wu, Y., \& Goldreich, P. 1999, ApJ, 519, 783 